\title{
Bicontinuous microemulsions for high yield, wet synthesis of ultrafine nanoparticles: a general approach $\uparrow$
}

\author{
Roman Latsuzbaia, Emanuela Negro and Ger Koper*
}

Received 12th January 2015, Accepted 29th January 2015

DOI: $10.1039 / c 5 f d 00004 a$

The design of a synthesis strategy for metal nanoparticles by templating dense microemulsions is proposed. Particle size is controlled by surfactant size rather than by microemulsion composition. The strategy was demonstrated with various systems with different surfactant: cationic, anionic and non-ionic and of different sizes. Formulations were determined using the microemulsion phase diagrams. Synthesis was demonstrated for platinum nanoparticles with some examples for gold. The nanoparticles were subsequently extracted from the microemulsion by absorption onto a carbon support, after which the surfactant was recycled.

\section{Introduction}

The great technological advancements that were promised with the introduction of nanomaterials can perhaps be achieved the most easily with the use of nanoparticles, as synthesis methods existed as long ago as Faraday's gold sol experiments, ${ }^{1}$ and knowledge of their applications in catalysis, optics and electronics is readily available. ${ }^{2-4}$ Most of the early synthesis procedures of metal nanoparticles consisted of coprecipitation of the water-soluble products from aqueous solution by mixing a suitable metal precursor with a reducing agent. ${ }^{5}$ The main synthesis techniques reported in the literature include co-precipitation, sol-gel processing, hydrothermal/ solvothermal, biomimetic and microemulsion approaches. ${ }^{5}$ However, as Wang et al. state in a recent review, ${ }^{6}$ despite the enormous amount of scientific papers and patents published in the field, actual understanding of the synthesis process appears to be still in its infancy. Nevertheless, insight is progressing quickly nowadays, as summarized in the same review, and it is very clear that aggregation plays a dominant role in most if not all synthesis procedures. ${ }^{6}$ Indeed, most existing synthesis methods only produce small and mono-dispersed nanoparticles at low concentrations. When higher yields and hence higher concentrations are required, particle

Department of Chemical Engineering, Delft University of Technology, Julianalaan 136, 2628 BL, Netherlands. E-mail: g.j.m.koper@tudelft.nl; Fax: +31 152784289

$\dagger$ Electronic supplementary information (ESI) available. See DOI: 10.1039/c5fd00004a 
sizes become uncontrollable and broad size distributions are obtained, ${ }^{7}$ largely as a result of aggregation. For a nanoparticle synthesis method to be attractive on a larger scale, routine production should not only have (1) a high yield of mono-dispersed nanoparticles of predefined size, but also use (2) a robust, yet flexible and safe production method with (3) an efficient and safe extraction technique.

Colloidal systems offer a great opportunity for the production of metal nanoparticles of predefined morphology, size and shape, using surfactants, organic ligands or polymers, which can stabilize the nanoparticles during the synthesis by preventing agglomeration. These systems act as a template and allow size reduction of synthesized nanoparticles down to a few nanometres $(<10 \mathrm{~nm}) .^{5,8} \mathrm{In}$ particular, synthesis in reverse micellar microemulsions was reported to have a high control over the nanoparticle size. ${ }^{8}$ These microemulsions are formed by self-assembly of the hydrophilic and hydrophobic phases or components into molecular interfaces, which result in various nano-structures such as micelles, bilayers, or continuous aqueous/oil nano-channels. ${ }^{9}$ These formed nano-structures can act as nano-reactors or templates for the synthesis of nanoparticles. Although reverse micellar systems offer control over size once the concentration of the nanoparticles in the system is increased, agglomeration is no longer prevented, ${ }^{10}$ and this significantly limits the yield of the nanoparticle production. This is not a problem in high surfactant content and dense (bi-)continuous microemulsions, which consist of local water and oil continuous phases or channels. ${ }^{11}$ It was previously shown by our group that monodisperse and small $(<5 \mathrm{~nm})$ platinum nanoparticles can be synthesized with a high yield in a sodium AOT/heptane/water (bi-)continuous microemulsion (BME). ${ }^{7}$ Here, we extend the previous results to a variety of surfactant systems and demonstrate the generality of the synthesis approach.

Interestingly, the theoretical background for the design method that we are about to present has been worked out quite recently, ${ }^{7,10,12,13}$ although this builds on much earlier results. ${ }^{14}$ It is the combination of these recent results, together with the novel understanding that under the optimal synthesis conditions it is the effective surfactant length that determines the nanoparticle size, that allowed us to formulate the present - almost quantitative - design method. As the most serious safety issues are concerned with the nanomaterial itself, ${ }^{15}$ the choice for a wet production route, together with deposition onto micron- or larger sized substrates in the final stage, as we advocate here, does satisfy contemporary criteria in the field. ${ }^{4}$

Hence, we report here on a general approach for the synthesis of small and uniform metal nanoparticles in dense microemulsions (Fig. 2). The method will be illustrated using various types of surfactants to control particle size in the nanometre range. The flexibility in the choice of surfactant allows us to employ up to $40 \mathrm{wt} \%$ water phase for synthesis, which is exceptionally high for microemulsion based synthesis and delivers high yields of the ultrafine nanoparticle synthesis (up to $3 \mathrm{wt} \%$ ).

\section{Experimental}

\section{Materials}

The chemicals used in this study have been purchased from Sigma-Aldrich unless otherwise specified and used without further purification. For the formation of 
microemulsions, the following surfactants have been used: dioctyl sodium sulfosuccinate, Na-AOT $\left(\mathrm{C}_{20} \mathrm{H}_{37} \mathrm{NaO}_{7} \mathrm{~S}, \geq 99 \%\right)$, didecyldimethylammonium bromide, DDAB $\left(\left[\mathrm{CH}_{3}\left(\mathrm{CH}_{2}\right)_{9}\right]_{2} \mathrm{~N}\left(\mathrm{CH}_{3}\right)_{2} \mathrm{Br}, 98 \%\right)$, hexadecyltrimethylammonium bromide, CTAB $\left(\mathrm{CH}_{3}\left(\mathrm{CH}_{2}\right)_{15} \mathrm{~N}(\mathrm{Br})\left(\mathrm{CH}_{3}\right)_{3}, 98 \%\right)$, Triton X-100 ( $t$-Oct- $\mathrm{C}_{6} \mathrm{H}_{4}{ }^{-}$ $\left.\left(\mathrm{C}_{2} \mathrm{H}_{4} \mathrm{O}\right)_{n} \mathrm{OH}, n=9-10\right)$ and Brij $\mathrm{L} 23\left(\mathrm{C}_{12} \mathrm{H}_{25}\left(\mathrm{C}_{2} \mathrm{H}_{4} \mathrm{O}\right)_{n} \mathrm{OH}, n \approx 23\right)$. The relevant surfactant structures are given in Fig. 1. Other components for the formation of various microemulsion systems heptane (anhydrous, 99\%), toluene (anhydrous, 99.8\%) and decane ( $\geq 99 \%)$ were obtained from Sigma-Aldrich; heptanol ( $\geq 99 \%)$ was purchased from Fluka. Water used in all our experiments was produced by a Milli-Q Ultra-Pure-Water system of Millipore-BV.

For the synthesis of platinum nanoparticles, hexachloroplatinic acid $\left(\mathrm{H}_{2} \mathrm{PtCl}_{6} \cdot 6 \mathrm{H}_{2} \mathrm{O}, 99.9 \%\right)$ and gold(III) chloride hydrate $\left(\mathrm{HAuCl}_{4}, 99.9 \%\right)$ were used as metal precursors. Sodium borohydride $\left(\mathrm{NaBH}_{4}\right.$, granular, $\left.\geq 98 \%\right)$ and hydrazine hydrate $\left(\mathrm{N}_{2} \mathrm{H}_{4}\right.$, water solution of $\left.25 \mathrm{wt} \%\right)$ were used as reducing agents. All reagents were purchased from Sigma-Aldrich. Vulcan XC-72R (E-TEK) was used for extraction of the nanoparticles from microemulsions. Tetrahydrofuran (THF) and acetone, both laboratory grade, were used for washing the nanoparticles supported on Vulcan XC-72R.

\section{Preparation of microemulsion and phase diagram study}

Five different surfactants were used in this work to form dense microemulsions for the synthesis of metal nanoparticles. When selecting the surfactant, its stability should be taken into account. For instance, Na-AOT is unstable and dimerizes when $\mathrm{N}_{2} \mathrm{H}_{4}$, a commonly used reducing agent, is used. Also, platinum nanoparticle formation does not happen with $\mathrm{Br}^{-}$containing surfactants, CTAB and $\mathrm{DDAB}$, due to anion exchange between the surfactant and the precursor, and the precise nature of the formed complexes is still unclear. ${ }^{\mathbf{1 6}}$

The synthesis procedure for all the systems reported in this work was as follows. Two microemulsions were prepared by vigorous mixing of the components and sonication for 15 minutes: one microemulsion contained a metal

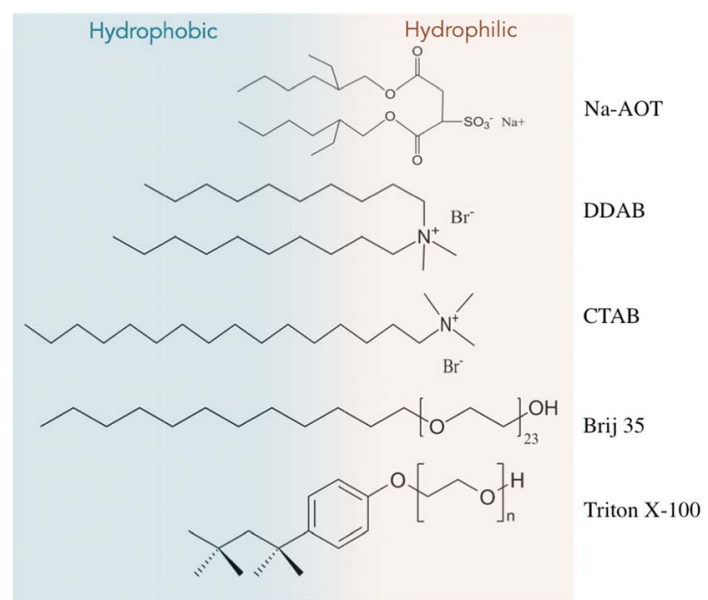

Fig. 1 Surfactants used for the synthesis of the metal nanoparticles. 
precursor in the aqueous phase, and the second microemulsion contained a ten times excess of the reduction agent. Afterwards the microemulsions are allowed to stabilize for one hour and gently mixed to form metal nanoparticles. The following microemulsions were used for the synthesis of the nanoparticles in this investigation (see the compositions in $\mathrm{ESI}^{\dagger}$ ): Na-AOT/heptane/water, Triton $\mathrm{X}$ 100/toluene/water, Brij 35/heptanol/water, DDAB/decane/water, CTAB/heptanol/ water and $\mathrm{CTAB} /$ heptane/water-butanol. A detailed description of the NP preparation and extraction is provided in the ESI. $\dagger$

\section{Characterization}

Electrical conductivity measurements were performed for the phase diagram studies of the microemulsions. Different electrical conductivity levels belong to differently nanostructured phases of the microemulsion. For these measurements, mixtures of various surfactant to oil ratios have been prepared and subsequently diluted with water to obtain microemulsions with the following mass fractions of water: $0.1,0.2,0.3,0.4,0.5,0.6,0.7,0.8$ and 0.9 . In this way the electrical conductivity was measured along the water dilution lines at constant surfactant to oil ratio. The electrical conductivity measurements were performed at $23{ }^{\circ} \mathrm{C}$ using a Metrohm 712 Conductometer with frequency 2.4 $\mathrm{kHz}$. The cell constant $\left(K=0.81 \mathrm{~cm}^{-1}\right)$ was calibrated using standard $\mathrm{KCl}$ solutions.

The size and morphology of the nanoparticles was also observed by Transmission Electron Microscopy (TEM). Two electron microscopes were used: FEI Monochromated Tecnai 200STEM-FEG operated at $200 \mathrm{kV}$, in which images were acquired with a Gatan CCD camera; Philips CM300UT-FEG point resolution 0.17 $\mathrm{nm}$, information limit: $0.1 \mathrm{~nm}$, operated at $200 \mathrm{kV}$, in which images were acquired with a TVIPS CCD camera. TEM measurements were carried out within 7 days after mixing the reactants. Specimens for TEM measurements were prepared by placing a single drop of the sample of Vulcan XC-72R supported platinum (or gold) nanoparticles dispersed in ethanol onto a Quantifoil R copper microgrid and subsequent drying for 24 hours in air. The size histograms of the nanoparticles were determined from at least 250 nanoparticles by image analysis (ImageJ) of TEM micrographs.

X-ray energy dispersive spectroscopy (EDS) coupled to TEM was employed to obtain elemental composition profiles of the samples. EDS in scanning transmission mode (STEM) with a spot size of $20 \mathrm{~nm}$ was used. No damage or transformation of the samples was observed during the measurement procedure. Additionally, scanning electron microscopy (SEM) and EDS measurements of the carbon-supported nanoparticles were performed on a JSM-6010LA microscope by JEOL. Prior to the measurement carbon supported nanoparticles were dispersed in ethanol by sonication ( 2 minutes), then a drop of the solution was deposited on a silicon wafer and dried.

Powder X-ray diffraction (XRD) patterns of the synthesized platinum nanoparticles were obtained with a diffractometer, from Bruker, equipped with Co-K $\alpha$ radiation $(\lambda=0.154 \mathrm{~nm})$. The measurements were carried out at $2 \theta=35-90^{\circ}$ $\left(0.014^{\circ}\right.$ step size $)$, with $1^{\circ}$ diverging and receiving slits at $50 \mathrm{kV}$ and $40 \mathrm{~mA}$. The XRD measurements were performed in order to verify the existence of the reduced platinum and its crystal structure. 


\section{Results and discussion}

Before the details of the method are presented and demonstrated, we briefly explain the requirements for a successful nanoparticle synthesis method and discuss the theoretical principles by which these can be achieved.

High yield in a wet synthesis process is the total mass of a nanomaterial produced per unit mass of liquid. For the precipitation reactions invoked here, the produced mass is equal to the amount of mass dissolved in the aqueous phase. Therefore, the larger the water fraction in the liquid, the larger the yield. Recently it was demonstrated by ourselves ${ }^{7}$ and by others ${ }^{17,18}$ that high yield production of mono-dispersed nanoparticles can be obtained by single phase, dense microemulsion systems. Microemulsions consist in their simplest form of water, oil and surfactant, but can have more ingredients such as co-surfactants. ${ }^{\mathbf{1 4}}$

In our synthesis approach, water continuity is required for the reactants to diffuse freely throughout the liquid. A dense nanostructure is required to prevent the aggregation and subsequent coalescence of the produced nanoparticles. Therefore, the synthesis yield was increased up to $3 \mathrm{wt} \%$, by increasing the concentrations of the metal precursor up to $500 \mathrm{mM}$ for Triton X-100 microemulsion, still no significant agglomeration occurred. In comparison, in droplet phase microemulsion synthesis the maximum reported metal concentration was $200 \mathrm{mM}^{7,19}$ The bicontinuous phase, where both the aqueous phase and the oil phase are continuous and form intertwining channels with monomolecular surfactant walls, is preferred because of its high level of nanostructure. Bicontinuous microemulsions form spontaneously when the surfactant is in its balanced state, where it has equal affinity for the oil phase as for the aqueous phase. ${ }^{14}$

Nanoparticle size in these bicontinuous microemulsions is controlled by the surfactant, ${ }^{10}$ as already outlined above. The surfactant packing parameter is defined as $v / a L$, where $v$ is the volume of the surfactant tail, $L$ is the effective surfactant length, and $a$ is the equilibrium area per molecule at the aggregate surface. A certain value of the surfactant parameter can be translated into a specific shape of the equilibrium aggregate by applying simple geometrical relations. ${ }^{14}$ The size of the aggregate is determined largely by the packing equation through the effective surfactant length. ${ }^{\mathbf{1 4 2 0}}$ The so-called surfactant packing equation $^{14}$ relates the surfactant parameter to the size of the nanoparticle, $R$, through the effective length of the surfactant, $L:^{21}$

$$
P=1+L / R+L^{2} /\left(3 R^{2}\right)
$$

The surfactant parameter has to be close to 1 , and preferably slightly larger to guarantee a continuous water phase. Hence, to a first approximation, the nanoparticle size becomes equal to the effective surfactant length. However, there are limits to consider: too small "amphiphilic" molecules such as small alcohols are not surface active. Too large molecules do not form microemulsions on a reasonable time scale: the molecules diffuse too slowly and the systems are arrested in long-lived mesophases. ${ }^{22}$

Monodispersity requires surfactant head group affinity towards the nanoparticle surface ${ }^{23}$ rather than to the aqueous phase. A strongly bound packing layer around the particle prevents further growth than the optimal size and at the 
same time protects it from binding to other nanoparticles. ${ }^{10} \mathrm{With}$ a loosely or nonbinding surfactant such a protective layer is not formed. A strong binding couple such as the sulphonate head group of Na-AOT and platinum guarantees the formation of very monodisperse nanoparticles, ${ }^{10}$ where the same surfactant with gold produces a qualitatively lesser result due to weaker interactions. Control of particle shape is expected for even stronger binding surfactants such that different crystal planes have significantly different binding affinities, ${ }^{24}$ but this is not the goal here.

Stability and reliability requires the components in the system, in particular the surfactant, to be inert with respect to the other chemicals used in the system. For instance, when hydrazine is used instead of borohydride as a reducing agent in the precipitation reaction, the surfactant dimerizes during the synthesis process, leading to unpredictable results. ${ }^{10}$

The design of a synthesis procedure that satisfies the given requirements can be carried out in a single series of 4 steps, graphically depicted in Fig. 2, although some optimization cycles may be necessary to attain the desired result. This will be illustrated with an example involving the synthesis of platinum nanoparticles as a catalyst for the oxygen reduction reaction, as takes place in a polyelectrolyte membrane fuel cell (Fig. S10†). To demonstrate some particular aspects, gold nanoparticle synthesis will also be discussed.

Selection of the surfactant is the obvious first step in the design, as there are three important conditions to be met. The first condition on the surfactant molecule is that it is to be in the balanced state, or, in other words, the surfactant parameter has to have a value of around 1 or slightly more to guarantee water continuity. ${ }^{\mathbf{1 4}}$ This corresponds to HLB-values (Hydrophile-Lypophile-Balance) of about 10 , typically between 8 and $12 .{ }^{14}$ The HLB values qualitatively describe the surfactant-water-oil equilibrium and account for molecular interactions of the surfactants with oil and water, and can be used to roughly estimate the packing parameter, $P \approx(20-\mathrm{HLB}) / 10,{ }^{25}$ although this can be estimated more precisely using the hydrophilic-lipophilic deviation (HLD). ${ }^{26}$ HLB-values for the various commercially available surfactants are typically provided by chemical suppliers. We found out that the composition of the microemulsion does weakly affect this value, but in a first design stage this may not be considered. The second condition on the surfactant is imposed by the effective surfactant length, $L$. Unfortunately, such values are generally not tabulated, however some can be found in the literature. A good first estimate can be obtained using Tanford's regression ${ }^{\mathbf{1 4}}$ involving the maximum chain length:

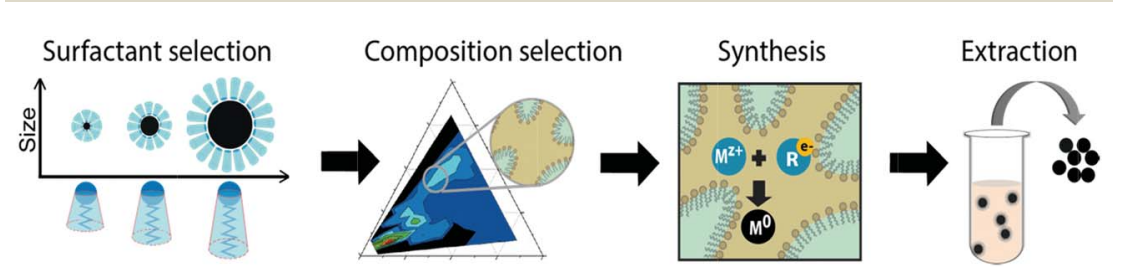

Fig. 2 A general approach to nanoparticle synthesis in dense microemulsion systems, consisting of four steps: (a) selection of surfactant, (b) determination of the phase diagram and selection of the microemulsion composition, (c) synthesis and (d) extraction of nanoparticles. 


$$
L \leq L_{\max } \approx(0.154+0.1265 n) \mathrm{nm},
$$

where $n$ is the number of carbon atoms. A third condition, affinity of the head group towards the metal surface and inertness with respect to the chemicals used, is a difficult one to predict, although some information is available in the literature. $^{27}$

For the present purpose of Pt and Au nanoparticle synthesis, we have selected various length surfactants with anionic (Na-AOT), cationic (DDAB and CTAB) and non-ionic (Triton X-100 and Brij 35) head groups, see Fig. 1 for additional information. All these molecules are frequently used for the synthesis of various types of nanomaterials including nanoparticles, so that ample characterization data is available. ${ }^{28}$ Nevertheless, they allow us to demonstrate the generality of the approach.

Formulation of the microemulsion composition is the logical next step. For the synthesis of the nanoparticles we employ high surfactant concentration or dense microemulsion systems. At high surfactant concentrations, (>30 wt\%) surfactants form locally oriented phases, such as spherical micelles in cubic packing, cylindrical micelles in hexagonal packing, and lamellar or bicontinuous structures. ${ }^{29}$ The formation of these structures depends on the curvature of the surfactantwater interface, which we can predetermine by carefully choosing the right surfactant based on its geometry. ${ }^{29}$ We aim for bicontinuous dense microemulsions, which can offer superior stability of the high concentrations of metal nanoparticles during the synthesis and enhanced reagent transport among the continuous water channels. ${ }^{\mathbf{1 0}}$

The oil type is largely dictated by the choice of the surfactant but some variation is usually possible. For instance, for Na-AOT one can choose alkanes from hexane to hexadecane, with the optimum being near heptane. ${ }^{10}$ This variation allows for some flexibility, for instance to fine-tune the particle size. The aqueous phase will be an electrolyte with an ionic composition similar to the expected liquid state after the precipitation reaction has completed. Using estimated values for the surfactant dimensions, one can now determine a theoretical phase diagram as proposed by Andre et al. ${ }^{\mathbf{2 0}}$ which involves limit lines below which a particular structure, e.g. cylinders packed in an interconnected face centered cubic (FCC) lattice, is geometrically possible. With this - or a more complete phase diagram in hand, one can determine the approximate position of the bicontinuous phase. ${ }^{7}$ Various techniques exist to refine the above described composition selection method as signatures of the bicontinuous phase are high conductivity, high viscosity, etc. $^{30}$

In Fig. 3 phase diagrams are presented for three systems involving Na-AOT, Triton X-100 and Brij 35. The colour scheme used is based on electrical conductivity, where regions of high and low conductivity are designated. Using a dilution line from the water corner through the approximate location of the bicontinuous phase, one can deduce the optimal composition from the measured conductivity values, see Table S3. $\dagger$

Synthesis of the nanoparticles is achieved by mixing two microemulsions, one with the reducing agent dissolved in the aqueous phase and the other with the dissolved metal precursor. Other schemes exist ${ }^{8}$ but will not be discussed here in detail. The reaction concludes typically within a few hours (Fig. S4-7†) and in principle it can be followed by UV-vis spectroscopy, although the particle 


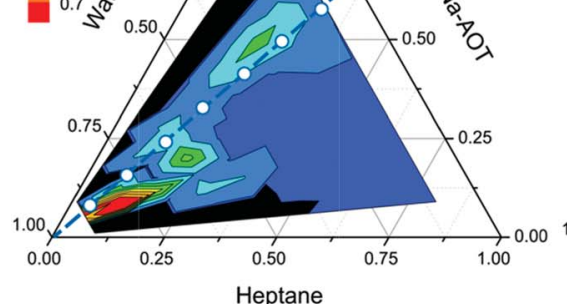

(a)

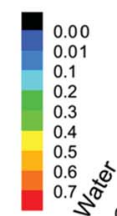

Paper

(c)

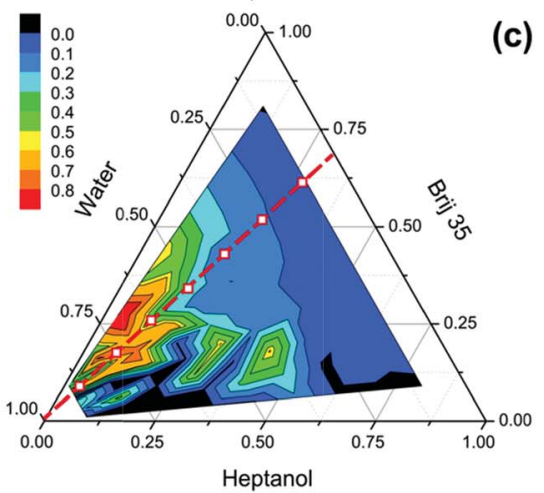

(b)

Fig. 3 Phase diagrams of microemulsions based on conductivity measurements $(a-c)$, colour maps based on relative electrical conductivity; white regions were not measured, and black regions represent phase-separated samples. Compositions are given as mass percentage. (d) Electrical conductivity along the dilution line of surfactant to oil ratio $7: 3$ for the microemulsions formed with Triton X-100 and Brij 35, and ratio $2: 1$ for Na-AOT microemulsion.

concentration very quickly becomes so high that the absorbance cannot be followed using a standard UV-vis spectrometer. ${ }^{\mathbf{1 0}}$ The resulting particles are usually studied by means of Transmission Electron Microscopy (TEM) on small aliquots of the produced liquid, strongly diluted to prevent as much as possible the immediate aggregation of the nanoparticles upon the breaking of the bicontinuous phase.

Fig. 4 gives results for the systems studied here for the synthesis of platinum and gold nanoparticles, more can be found in the ESI. $\dagger$ The correlation of nanoparticle size with surfactant size is clear (Table 1); as predicted it was the main parameter to control the nanoparticle size. The difference in size from Au to Pt for the same surfactant is to be attributed to the differences in head group binding to the metal affecting surfactant conformation. Binding to Pt is strong for Na-AOT and Triton X-100 so that small and monodisperse nanoparticles with Relative Standard Deviation (RSD) of $17 \%$ and $22 \%$ are obtained. This is not the case for Brij 35, which has a large hydrophilic part and a short hydrophobic part that do not allow steric stabilization, so polydisperse nanoparticles are formed 

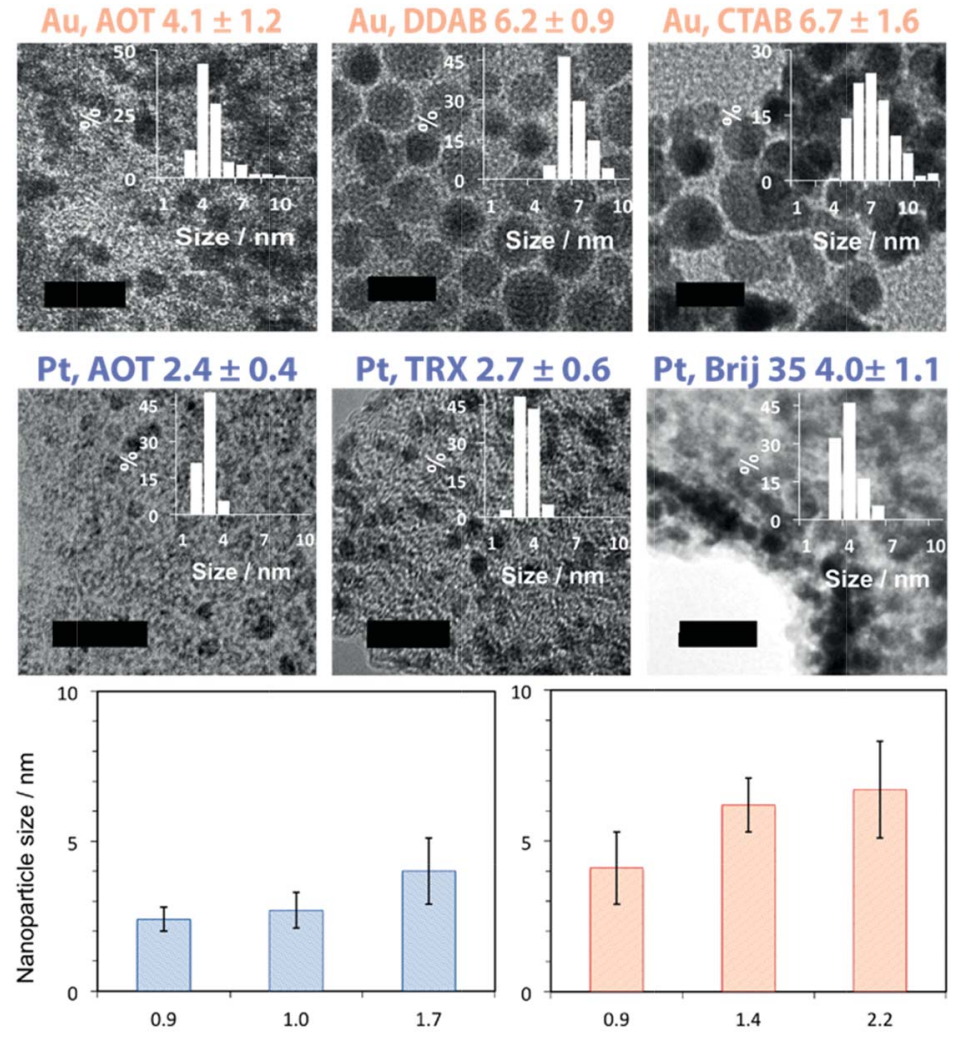

Maximum surfactant chain length / $\mathrm{nm}$

Fig. 4 Size dependence of nanoparticles of platinum (in blue) and gold (in orange) on the surfactant used to form the templating microemulsion. Scale bar $10 \mathrm{~nm}$.

(RSD 28\%). In the case of gold, ionic surfactants can stabilize nanoparticles for at least a week but non-ionic surfactants, e.g. Triton X-100, nanoparticles, grow in size starting from $<10 \mathrm{~nm}$ and growing within an hour to over $50 \mathrm{~nm}$. This is observed as a color change of the nanoparticle containing microemulsion from red to purple (Fig. S7, ESI $\dagger$ ). Here the surfactant does not control the size, although it can be controlled by reaction time. An undesired reactivity effect is the difficulty of platinum nanoparticle formation with $\mathrm{Br}^{-}$containing surfactants, due to the formation of platinum bromide complexes with different reactivity from the chlorides $;^{16}$ with gold this is not an issue.

No other parameters significantly influence the size of nanoparticles in these dense microemulsion systems. Various microemulsion compositions or reagent concentrations were used for the synthesis of nanoparticles in the same system, which did not result in a different nanoparticle size, proving the robustness of the method (Fig. S13-15†). ${ }^{10}$ The method allows synthesis of various metals such as Pd (Fig. S-16†), Fe, Co, Ni and metal alloys such as Pt-Ru-Co (Fig. S17†), also previously reported by us for the Na-AOT system. ${ }^{7}$

Extraction methods that can be employed strongly depend on what application the nanoparticles will later be required for. For catalysis, adsorption of 
Table 1 Relevant parameters of the surfactants used in this work and the resulting NP sizes

\begin{tabular}{|c|c|c|c|c|c|}
\hline Surfactant & $\begin{array}{l}\text { Number of } \\
\text { hydrocarbons } n\end{array}$ & $\begin{array}{l}\text { Maximum } \\
\text { chain length }^{b} \\
L_{\max }[\mathrm{nm}]\end{array}$ & $\begin{array}{l}\text { Surfactant } \\
\text { packing } \\
\text { parameter }^{c} P\end{array}$ & $\begin{array}{l}\text { Diameter } \\
\text { of Pt NPs } \\
D[\mathrm{~nm}]\end{array}$ & $\begin{array}{l}\text { Diameter } \\
\text { of Au NPs } \\
D[\mathrm{~nm}]\end{array}$ \\
\hline Na-AOT & 6 & 0.9 & 1.2 & $2.4 \pm 0.4$ & $4.1 \pm 1.2$ \\
\hline Triton X-100 & $4^{a}$ & 1.0 & 0.8 & $2.7 \pm 0.6$ & - \\
\hline DDAB & 10 & 1.4 & 1.1 & - & $6.2 \pm 0.9$ \\
\hline Brij 35 & 12 & 1.7 & 0.6 & $4.0 \pm 1.1$ & - \\
\hline CTAB & 16 & 2.2 & 1.0 & - & $6.7 \pm 1.6$ \\
\hline
\end{tabular}

${ }^{a}$ Contains one aromatic ring in the hydrophobic tail. ${ }^{b}$ Maximum chain length defined in ESI and roughly equal to the effective length of the surfactant. ${ }^{c}$ Effective packing parameters for Na-AOT, DDAB and CTAB are available from ref. 20, 31 and 32, whereas for the other surfactants the packing parameter was estimated from the HLB value.

the nanoparticles onto a carrier is the preferred method. ${ }^{4}$ After binding the nanoparticles to the substrate, these can easily be separated from the production liquid, which then becomes available for recycling: only the remainder of the salt needs to be extracted from the aqueous phase. No heat treatment with associated nanoparticle agglomeration (Fig. S11†) or surfactant decomposition is required. After adsorption onto the carbon support, the surfactant was removed by washing and the platinum surface was clean for catalysis (Fig. S10†).

We succeeded in harvesting nanoparticles and achieved a uniform surface coverage on a carbon support due to hydrophobic interactions of the surfactant aliphatic chains and hydrophobic support. ${ }^{33}$ However, depending on the nature of the surfactant, various dispersion levels could be achieved: Triton X-100 and Na-AOT facilitate good surface coverage, whereas with Brij 35 clustering occurs due to the large hydrophilic domains of the surfactant molecule, see Fig. 5 and $\mathrm{S} 12 . \dagger$
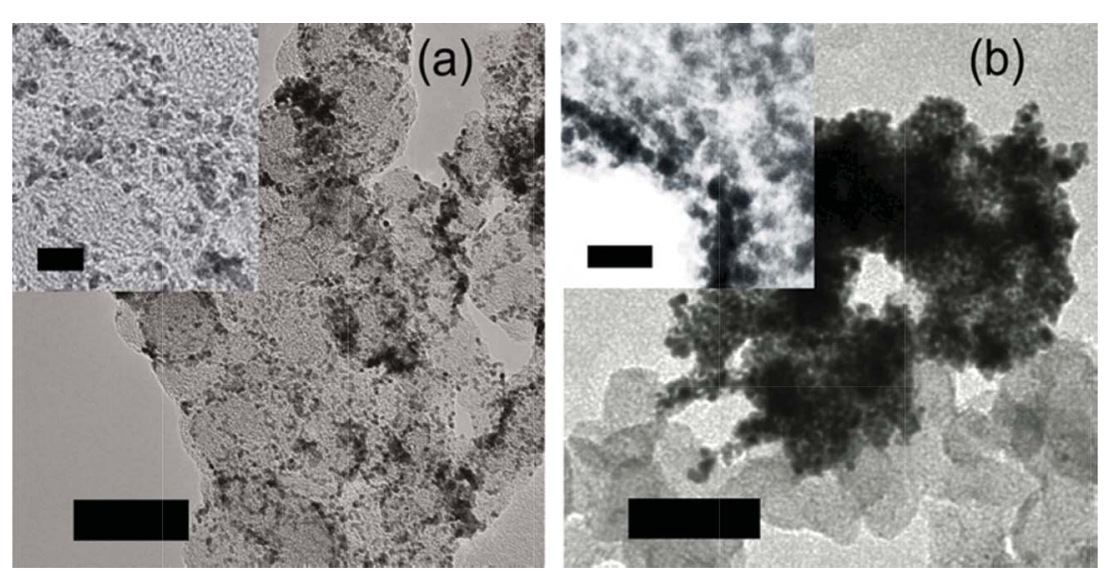

Fig. 5 Coverage of Pt nanoparticles on carbon support showing (a) excellent dispersion for Triton X-100, and (b) agglomeration for Brij 35. Scale bar 50 nm, inset scale bar $10 \mathrm{~nm}$. 


\section{Conclusions}

In summary, we have demonstrated that a robust synthesis route is available for the wet synthesis of uniformly sized various metal nanoparticles, which is scalable yet flexible for further optimization. The dense microemulsion system allows a change of reactant concentration, microemulsion composition to increase the yield of the process, while none of these parameters influences the final size of the nanoparticles. The process design and system selection procedure are theoretically underpinned by considering the surfactant parameter, the effective surfactant size, the microemulsion phase diagram and the influence of formulation variables. Remarkable yields up to $3 \%$ in weight were achieved (Table S2 $\uparrow$ ), as compared to a $0.4 \%$ maximum yield for micellar systems. ${ }^{7}$

\section{Acknowledgements}

This work was supported by the Ministry of Economic Affairs of the Netherlands in the framework of IOP-Self Healing Materials (SHM) Program and the COST CM1101 Action. We thank Louw Florusse and Vincent le Sage for TEM measurements, Elena Galan Garcia for NMR and students: L. Baas, P. Carter, T. Vermunt and M. van der Meij for the phase diagram studies.

\section{References}

1 M. Faraday, Philos. Trans. R. Soc. London, 1857, 147, 145-181.

2 Y. Xia, Y. Xiong, B. Lim and S. E. Skrabalak, Angew. Chem., Int. Ed., 2009, 48, 1.

3 G. Schmid, in Nanoparticles, Wiley-VCH Verlag GmbH \& Co. KGaA, 2005, pp. 1-3.

4 D. Astruc, F. Lu and J. R. Aranzaes, Angew. Chem., Int. Ed., 2005, 44, 7852-7872.

5 B. L. Cushing, V. L. Kolesnichenko and C. J. O'Connor, Chem. Rev., 2004, 104, 3893-3946.

6 F. Wang, V. N. Richards, S. P. Shields and W. E. Buhro, Chem. Mater., 2013, 26, 5-21.

7 K. Kowlgi, U. Lafont, M. Rappolt and G. Koper, J. Colloid Interface Sci., 2012, 372, 16-23.

8 S. Eriksson, U. Nylén, S. Rojas and M. Boutonnet, Appl. Catal., A, 2004, 265, 207-219.

9 D. Chandler, Nature, 2005, 437, 640-647.

10 E. Negro, R. Latsuzbaia and G. J. M. Koper, Langmuir, 2014, 30, 8300-8307.

11 P. Guering and B. Lindman, Langmuir, 1985, 1, 464-468.

12 P. J. Krommenhoek, J. Wang, N. Hentz, A. C. Johnston-Peck, K. A. Kozek, G. Kalyuzhny and J. B. Tracy, ACS Nano, 2012, 6, 4903-4911.

13 M. A. López-Quintela, Curr. Opin. Colloid Interface Sci., 2003, 8, 137-144.

$14 \mathrm{~J}$. N. Israelachvili, in Intermolecular and Surface Forces, ed. J. N. Israelachvili, Academic Press, San Diego, 2011, pp. 535-576.

15 Y. F. Li and C. Chen, Small, 2011, 7, 2965-2980.

16 E. M. Glebov, A. V. Kolomeets, I. P. Pozdnyakov, V. F. Plyusnin, V. P. Grivin, N. V. Tkachenko and H. Lemmetyinen, RSC Adv., 2012, 2, 5768-5778.

17 J. Esquivel, I. Facundo, M. E. Treviño and R. López, J. Mater. Sci., 2007, 42, 9015-9020. 
18 J. A. E. Pamela, Y. Reyes, M. E. Treviño, H. Saade and R. G. López, J. Nanomater., 2010, 2010.

19 D.-H. Chen, J.-J. Yeh and T.-C. Huang, J. Colloid Interface Sci., 1999, 215, 159166.

20 P. Andre, B. W. Ninham and M. P. Pileni, New J. Chem., 2001, 25, 563-571.

21 W. Kunz, F. Testard and T. Zemb, Langmuir, 2009, 25, 112-115.

22 G. Lagaly, W. Richtering and K.-G. Hauptversammlung, Mesophases, Polymers, and Particles, Springer, 2004.

23 B. Nikoobakht and M. A. El-Sayed, Langmuir, 2001, 17, 6368-6374.

24 Y. Xia, Y. Xiong, B. Lim and S. E. Skrabalak, Angew. Chem., Int. Ed., 2009, 48, 60-103.

25 P. Becher, J. Dispersion Sci. Technol., 1984, 5, 81-96.

26 E. Acosta, J. Yuan and A. Bhakta, J. Surfactants Deterg., 2008, 11, 145-158.

27 M. J. Rosen and J. T. Kunjappu, Surfactants and Interfacial Phenomena: Fourth Edition, 2012.

28 M. A. López-Quintela, C. Tojo, M. C. Blanco, L. García Rio and J. R. Leis, Curr. Opin. Colloid Interface Sci., 2004, 9, 264-278.

29 I. W. Hamley, Angew. Chem., Int. Ed., 2003, 42, 1692-1712.

30 A. Bumajdad and J. Eastoe, J. Colloid Interface Sci., 2004, 274, 268-276.

31 S. T. Hyde, B. W. Ninham and T. Zemb, J. Phys. Chem., 1989, 93, 1464-1471.

32 M. Giustini, G. Palazzo, G. Colafemmina, M. Della Monica, M. Giomini and A. Ceglie, J. Phys. Chem., 1996, 100, 3190-3198.

33 K. Yurekli, C. A. Mitchell and R. Krishnamoorti, J. Am. Chem. Soc., 2004, 126, 9902-9903. 